\title{
RELACIÓN ENTRE ESTILOS DE APRENDIZAJE Y RENDIMIENTO ESCOLAR EN FÍSICA Y QUÍMICA DE SECUNDARIA
}

Felipe Quintanal-Pérez1: Colegio marista La Inmaculada. España felipeqp@maristasmediterranea.com

\section{RESUMEN}

1 presente artículo pone de manifiesto la relación existente entre los diferentes estilos de aprendizaje y el rendimiento académico en la Física y Química de $4^{\circ}$ de Secundaria. Para ello se determinaron los estilos de aprendizaje de los alumnos de cuatro centros concertados andaluces, así como sus calificaciones escolares. Se intervino en uno de los centros empleando diversas estrategias de enseñanza y se comprobó su incidencia en el rendimiento escolar de los alumnos. Por último, se buscaron qué estilos se asociaban a rendimientos escolares elevados y cuáles a rendimientos insatisfactorios.

PALABRAS CLAVE: Estilos de Aprendizaje - Rendimiento académico - CHAEA Física y Química.

\section{RELATIONSHIP BETWEEN LEARNING STYLES AND STUDENT PERFORMANCE IN PHYSCIS AND CHEMISTRY OF SECONDARY SCHOOL}

\begin{abstract}
This article highlights the relationship between learning styles and academic performance in Physics and Chemistry 4 th High School. We identified the learning styles of students in four Andalusian aided schools and their school grades. It was operated at one of the schools using various teaching strategies and found its impact on students' academic performance. Finally, we looked for what styles were associated with higher academic performance and what to unsatisfactory performance.

\footnotetext{
${ }^{1}$ Autor correspondiente

Felipe Quintanal-Pérez: Doctor Profesor de Física y Química de Secundaria y Bachillerato. Colegio marista La Inmaculada. Granada, España.

Correo: felipeqp@maristasmediterranea.com
} 
KEY WORDS: Learning Styles - Student Performance - HALSQ - Physics and Chemistry.

\section{INTRODUCCIÓN}

\subsection{Origen de la investigación}

El origen de esta investigación se ancla en dos raíces poderosas. La primera recoge las lecturas previas efectuadas que han suscitado una serie de nuevas preguntas ynuestro interés por generar nuevas ideas. La segunda ha sido nuestra propia experiencia, que nos ha permitido conocer el contexto en el que se desarrollan los hechos educativos sobre los que queríamos indagar y obtener una serie de respuestas a los problemas detectados en la praxis.

En este caso, se deben destacar los siguientes factores: la inquietud personal de iniciar investigaciones en torno a la formación en Internet como ámbito que ofrece nuevas posibilidades de aprendizaje y, por tanto, cómo y en qué circunstancias se mejora el aprendizaje; las metodologías de enseñanza, ya que desde hace varios años, se cuestiona la ciencia escolar, sus resultados y el interés desarrollado hacia ella; el cariño personal del autor hacia el campo de la Física y Química y, por último, aunque no menos importante, el deseo personal de contribuir al desarrollo de estudios e iniciativas formativas en los nuevos espacios educativos.

\subsection{Fundamentación y contexto de la investigación}

La fundamentación de la investigación efectuada se asienta en el hecho de que el principal problema que la enseñanza, el aprendizaje de la Ciencia en la escuela y la investigación en Didáctica de la Ciencia deben afrontar hoy son las actitudes inadecuadas y de repulsa de los estudiantes hacia la Ciencia, y más específicamente, la falta de interés hacia la Ciencia en la escuela.

Este diagnóstico de carácter negativo presenta, además, un perfil temporal variable definido, según multitud de investigaciones, por un mínimo muy acusado en el período de la adolescencia temprana. Este progresivo hundimiento en el interés de los adolescentes por la Ciencia se agudiza en el caso de las chicas, por lo que se produce un alejamiento de la ciencia escolar y el consiguiente abandono de las opciones o carreras científicas una vez que se presentan éstas en los diferentes periplos académicos seguidos por los alumnos.

Además, influyen otra serie de variables intermedias. A la ya citada del género se debe destacar la percepción que se tiene sobre cada una de las ciencias que se trate. Así, no es lo mismo hablar de Matemáticas, Física o Química que de Biología, Tecnología o Informática. Destacar también la incidencia de factores culturales en el propio país, el profesor, la falta de trabajo de laboratorio o la excesiva inclinación de las clases para preparar exámenes. 
Por ello, el interrogante que fundamenta la investigación efectuada se enmarca en el contexto de centros concertados al desarrollar el investigador su labor docente en este ámbito.

Para cerrar el ciclo, la investigación se centró en el Rendimiento Escolar de Física y Química en $4^{\circ}$ de Educación Secundaria Obligatoria (ESO), ya que periódicamente, los diversos medios de comunicación nos alertan del escaso rendimiento de los alumnos españoles en esta materia y atacan a todo lo que se pone delante: profesorado, sistemas de enseñanza, metodologías, currículos, políticas educativas, administraciones locales, autonómicas y gubernamentales, etc.

Resulta interesante, sin embargo, señalar que diversos informes manifiestan que el campo científico es un espacio valorado e interesante para los jóvenes. No obstante y de forma lamentable, dicho interés no se trasluce en un rendimiento académico positivo en las asignaturas de ciencias.

Parece relevante señalar que ello puede estar motivado por diversas causas, de las que destacamos el bajo nivel del estudiantado en las disciplinas científicas, el clasicismo de gran parte del profesorado de estas asignaturas y el carácter propedéutico que se les concede. Todo ello convierte a las materias científicas y, en particular, a la Física y Química en disciplinas monótonas, rutinarias, aburridas y carentes de interés para la vida diaria.

\subsection{Marco conceptual: Los Estilos de Aprendizaje y la Física y Química}

Inicialmente se encuadró la investigación dentro del marco del aprendizaje del alumnado y, más concretamente, dentro de los Estilos de Aprendizaje de éstos. Para ello se efectuó una revisión bibliográfica amplia sobre las nociones diversas de estilo, las diferentes teorías del aprendizaje y los diversos conceptos relacionados con el término Estilos de Aprendizaje.

La definición aceptada para esta investigación responde a la consideración de que los Estilos de Aprendizaje "son los rasgos cognitivos, afectivos y fisiológicos, que sirven como indicadores relativamente estables, de cómo los discentes perciben, interaccionan y responden a sus ambientes de aprendizaje" 2

La Dra. Alonso ${ }^{3}$, basándose en los resultados de su investigación, elaboró una lista con las características principales de cada estilo:

a)

Activo:

animador,

improvisador, descubridor, arriesgado, espontáneo.

\footnotetext{
2 Alonso, C., Gallego, D.\& Honey, P. (2006). Los Estilos de Aprendizaje. Procedimientos de Diagnóstico y Mejora Mensajero. Bilbao.

${ }^{3}$ Alonso, C., Gallego, D.\& Honey, P. (2006). Los Estilos de Aprendizaje. Procedimientos de Diagnóstico y Mejora. Mensajero. Bilbao
} 
b) concienzudo, receptivo, analítico, exhaustivo.

c) objetivo, crítico, estructurado.

d) experimentador, práctico, directo, eficaz, realista.

Reflexivo: ponderado, Teórico: metódico, lógico, Pragmático:

Las características de las personas con predominancia en cualquiera de los Estilos de Aprendizaje se pueden definir de la siguiente manera:

a) Estilo Activo: Se implican plenamente y sin prejuicios en nuevas experiencias. Son de mente abierta, nada escépticos y acometen con entusiasmo las tareas nuevas. Son gentes del aquí y ahora y les encanta vivir nuevas experiencias. Sus días están llenos de actividad. Piensan que por lo menos una vez hay que intentarlo todo. Tan pronto como desciende la excitación de una actividad, comienzan a buscar la próxima. Se crecen ante los desafíos que suponen nuevas experiencias, y se aburren con los largos plazos. Son personas muy de grupo que se involucran en los asuntos de los demás y centran a su alrededor todas las actividades.

b)

considerar las experiencias y observarlas desde diferentes perspectivas. Reúnen datos, analizándolos con detenimiento antes de llegar a alguna conclusión. Su filosofía consiste en ser prudente, no dejar piedra sin mover, mirar bien antes de pasar. Son personas que gusta considerar todas las alternativas posibles antes de realizar un movimiento. Disfrutan observando la actuación de los demás, escuchan a los demás y no intervienen hasta que se han adueñado de la situación.

c)

Estilo Teórico: Adaptan e integran las observaciones dentro de teorías lógicas y complejas. Enfocan los problemas de forma vertical escalonada, por etapas lógicas. Tienden a ser perfeccionistas. Integran los hechos en teorías coherentes. Les gusta analizar y sintetizar. Son profundos en su sistema de pensamiento, a la hora de establecer principios, teorías y modelos. Para ellos, si es lógico es bueno. Buscan la racionalidad y la objetividad huyendo de lo subjetivo y de lo ambiguo.

d)

fuerte de estas personas es la aplicación práctica de las ideas. Descubren el aspecto positivo de las nuevas ideas y aprovechan la primera oportunidad para experimentarlas. Les gusta actuar rápidamente y con seguridad con aquellas ideas y proyectos que les atraen. Tienden a ser impacientes cuando hay personas que teorizan. Pisan tierra cuando hay que tomar una decisión o resolver un problema. Su filosofía es siempre se puede hacer mejor, si funciona es bueno.

El vínculo que puede relacionar Estilos de Aprendizaje y Física y Química nos empuja a acercarnos a la Didáctica de estas disciplinas. Por ello, en la investigación 
efectuada nos aproximamos a los recursos más empleados por el profesorado y a la percepción que tienen de éstos los alumnos. Dichos recursos fueron las sesiones teóricas, las prácticas de laboratorio y los seminarios. La conclusión fue clara; influyen los diversos estilos de enseñanza del profesorado que, a su vez, están condicionados por sus propios Estilos de Aprendizaje.

La literatura científica es amplia en constatar el hecho de que, en general, el profesorado de Física y Química es renuente a la hora de modificar la impartición de sus clases, a pesar de las diferentes reformas educativas y la aparición de nuevos modelos de enseñanza. Múltiples factores avalan este hecho, de los cuales entresacamos los modelos de enseñanza, los Estilos de Aprendizaje usados por los profesores, los factores culturales de éstos, los modelos de aprendizaje de los discentes, las ideas del profesor sobre lo que es la materia impartida y la Ciencia en general y las teorías motivacionales.

Por ello, es fundamental ensamblar los estilos de enseñanza de los profesionales con los Estilos de Aprendizaje de los estudiantes, de forma que los primeros favorezcan y mejoren todos los estilos de los segundos.

\subsection{Los Estilos de Aprendizaje y el Rendimiento Escolar}

Es complicado aproximarse al concepto de Rendimiento Escolar o Académico, pues la inmensa mayoría de los investigadores, los profesionales de la enseñanza, los alumnos y el público en general lo asocia a las calificaciones escolares obtenidas por los estudiantes en las diversas asignaturas. No obstante, dicho concepto es más rico y pluriforme de lo que cabría esperar inicialmente y se encuentra influido por multitud de variables.

Cabe agrupar a dichas variables en personales y contextuales. Las variables personales engloban a la inteligencia, aptitudes, Estilos de Aprendizaje, conocimientos previos, género, edad, autoconcepto y metas de aprendizaje. Las variables contextuales, por su parte, abarcan el status socioeconómico y cultural de las familias, la escuela como institución educativa, los contenidos académicos, los métodos de enseñanza, las tareas escolares, las expectativas de los profesores y las de los estudiantes.

De todos los factores señalados es particularmente relevante para la investigación efectuada la relación existente entre Estilos de Aprendizaje y Rendimiento Escolar. Múltiples investigaciones e informes apoyan el hecho de que el Rendimiento Académico se incrementa cuando la enseñanza empleada se ajusta a los Estilos de Aprendizaje de los discentes. Ello no descarta que haya que fomentar la habilidad de los estudiantes en cambiar de Estilo de Aprendizaje según lo requieran las circunstancias y un entrenamiento de la capacidad del profesorado en el uso de los diferentes Estilos de Enseñanza y de Aprendizaje con objeto de poder ayudar a todo su alumnado. 
La investigación realizada tuvo en cuenta estas consideraciones y propuso tres estrategias concretas para favorecer el aprendizaje: el uso de las TIC, el empleo de la Ciencia Recreativa y la realización de una Semana de la Ciencia y Tecnología, con objeto de influir en los Estilos de Aprendizaje de los alumnos y contribuir a un mejor Rendimiento Académico de éstos en la asignatura de Física y Química.

\subsection{Objetivos}

El objetivo fundamental se formuló como sigue: Analizar los Estilos de Aprendizaje de los alumnos de $4^{\circ}$ de ESO de los centros de capitales andaluzas de la Provincia Mediterránea de la Institución marista, el predominio de determinados estilos, su incidencia y la mejora en el Rendimiento Académico de la Física y Química.

a) acompañó de los siguientes objetivos específicos:

b) Aprendizaje de los alumnos de $4^{\circ}$ de ESO en los centros de capitales andaluzas de la Provincia Mediterránea.

c)

Estilos de Aprendizaje preferenciales.

d) preferidos convergen o divergen en los centros investigados.

e) preferenciales suponen una base para la enseñanza de la Física y Química.

f) uso de determinadas estrategias de enseñanza - aprendizaje y el predominio de determinados estilos en el Rendimiento Académico de la Física y Química.

\section{METODOLOGÍA}

\subsection{Metodología de la investigación empírica}

Se plantearon las siguientes hipótesis de trabajo:

a) un perfil de preferencias único de Estilos de Aprendizaje en los alumnos de $4^{\circ}$ de ESO investigados.

b) preferencias de los Estilos de Aprendizaje no se encuentran condicionadas de manera clara por el Estilo Educativo de la Institución educativa.

c)

Hipótesis 3: La ciudad de origen de los alumnos influye ligeramente, pero no de manera significativa en los Estilos de Aprendizaje de los alumnos. 
d)

Hipótesis 4: La vigorización de los diversos Estilos de Aprendizaje influye en el Rendimiento Académico de la Física y Química.

e)

Hipótesis 5: No existen diferencias apreciables estadísticamente entre los Estilos de Aprendizaje en relación a determinadas variables socioculturales como el sexo, la edad, el año académico o la zona de residencia.

Como variables dependientes del estudio se establecieron la preferencia de Estilos de Aprendizaje y el Rendimiento Académico de la Física y Química, mientras que como variables independientes se situó el curso de cuarto de ESO y el Estilo Educativo de la institución educativa.

La muestra escogida estuvo formada por 263 alumnos de cuarto de ESO de los centros de Córdoba, Granada, Jaén y Sevilla de la Provincia Mediterránea; el tipo de muestra escogida fue el de muestra invitada y el muestreo, aleatorio, bietápico.

Las técnicas e instrumentos empleados fueron la encuesta para la determinación de la preferencia de Estilos de Aprendizaje de alumnos y profesores y las calificaciones escolares de Física y Química en junio de 2009 para medir el Rendimiento Académico. Dentro de la técnica encuesta se seleccionó como instrumento el cuestionario y entre éstos se eligió el Cuestionario Honey - Alonso de Estilos de Aprendizaje (CHAEA).

El CHAEA consta de 80 ítems y tiene presente cuatro Estilos de Aprendizaje fundamentales: estilo Activo, estilo Reflexivo, estilo Teórico y estilo Pragmático. A cada estilo le corresponden 20 ítems, distribuidos al azar y cada encuestado debe contestar (+) o (-) según sus respuestas se aproximen con mayor o menor fidelidad a los enunciados expuestos en cada ítem.

\subsection{Metodología de la investigación complementaria}

Se basó en la aplicación de tres estrategias de enseñanza - aprendizaje en la asignatura de Física y Química de $4^{\circ}$ de ESO en un centro específico, denotado como Centro 2, para fortalecer los Estilos de Aprendizaje de sus alumnos.

La primera estrategia, uso de aplicaciones TIC, consistió en la generación de una serie de protocolos para que los alumnos pudiesen trabajar con determinadas aplicaciones informáticas que estaban ubicadas en diversos portales de Internet. Cada una de estas utilidades potenciaba determinados Estilos de Aprendizaje y favorecían el repaso de la materia impartida, así como su consolidación. Al carecer de ordenadores en las clases, se dispuso una hora de la asignatura conectada con otra en la que el aula de Informática se encontraba disponible.

La segunda estrategia, empleo de la Ciencia Recreativa, se basaba en la utilización de juguetes científicos, pequeñas experiencias caseras o que requiriesen poco material de laboratorio para reforzar los diferentes Estilos de Aprendizaje de los estudiantes, 
incitar su curiosidad, desplegar su capacidad de emitir hipótesis, establecer algún tópico o robustecerlo.

La tercera estrategia, realización de una Semana de la Ciencia, consistió en la secuenciación de una serie de actividades aplicadas a los alumnos de $4^{\circ}$ de ESO en las postrimerías del curso escolar.

Se realizaron conferencias magistrales, talleres de Física Cotidiana, de Divulgación de la Ciencia, de Cine Científico, exposiciones y una final que agrupó a los mejores proyectos científicos elaborados, presentados y escogidos por los alumnos. Se concluyó efectuando una encuesta, no validada, concerniente a la percepción y satisfacción de los estudiantes sobre la Semana de la Ciencia, que corroboró la aceptación y agrado que había dejado dicha actividad.

\section{ANÁLISIS Y DISCUSIÓN}

\subsection{Perfil del profesorado de la asignatura}

Los profesores de Física y Química de los Centros estudiados, en total cuatro, presentaron predilección por los estilos Reflexivo y Teórico, aunque se observó una diferencia clara en cuanto a los estilos Activo y Pragmático que también aparecieron desarrollados para los profesores de los Centros 2 y 3 y con menos predilección para los docentes de los Centros 1 y 4.

\subsection{Resultados estadísticos descriptivos obtenidos por los alumnos con el cuestionario CHAEA}

Los alumnos que respondieron al Cuestionario CHAEA fueron 263, de los cuales 68 pertenecían al Centro 1, 55 al Centro 2, 70 al Centro 3 y 70 al Centro 4. Por género, 221 eran varones y 42 mujeres. Por edad, el 49.42\% tenía 15 años, el $46 \%$ era de 16 años y el 4.56\% presentaba 17 o más años. Los valores obtenidos en Estilos de Aprendizaje se muestran en la Tabla 1

Tabla 1. Estadística descriptiva de los Estilos de Aprendizaje.

\begin{tabular}{|c|c|c|c|c|}
\hline \multicolumn{5}{|c|}{ MUESTRA COMPLETA } \\
\hline N = 263 & ACTIVO & REFLEXIVO & TEÓRICO & PRAGMÁTICO \\
\hline MEDIA & 12,76 & 13,61 & 12,91 & 13,40 \\
\hline $\begin{array}{c}\text { DESVIACIÓN } \\
\text { TÍPICA }\end{array}$ & 3,41 & 3,10 & 3,09 & 2,76 \\
\hline VARIANZA & 11,62 & 9,59 & 9,53 & 7,62 \\
\hline MÍNIMO & 1 & 1 & 1 & 1 \\
\hline MÁXIMO & 20 & 20 & 20 & 20 \\
\hline
\end{tabular}


De estos resultados se deduce que los alumnos presentaron preferencias moderadas en los cuatro Estilos de Aprendizaje, aunque hay una ligera tendencia hacia los estilos Reflexivo, Pragmático, seguidos del Teórico y del Activo. Por género, los resultados obtenidos se representan mediante la Tabla 2.

Tabla 2. Estilos de Aprendizaje por género. Fuente: Elaboración propia

\begin{tabular}{cccccc}
\hline Género & N & Activo & Reflexivo & Teórico & Pragmático \\
\hline Masculino & 212 & 12,53 & 13,59 & 12,86 & 13,25 \\
Femenino & 42 & 13,98 & 13,69 & 13,19 & 14,17 \\
\hline
\end{tabular}

De la Figura 2 se infiere que las medias obtenidas por las alumnas fueron superiores en todos los estilos a las de los alumnos.

Este análisis se repitió para cada Centro, considerado en su totalidad y separadamente para cada sexo, en este apartado y en los posteriores. Se dedujo que las mejores medias, obtenidas en conjunto, correspondieron al Centro 3 y las peores al Centro 2, centro de nuestra intervención. Parece obvio señalar que, al igual que ocurría con los profesores, medias altas en estilos Reflexivo y Teórico permiten esperar rendimientos altos en Física y Química; por tanto, cabía suponer que el rendimiento escolar de esta materia debía ser muy alto en el Centro 3 y escaso en el Centro 2.

\subsection{Combinaciones de Estilos de Aprendizaje con preferencias alta/muy alta}

En la muestra constituida por todos los Centros destacaron las combinaciones con preferencias alta / muy alta del estilo Activo. Se mostró en las formas activa - teórica, activa - pragmática, activa - reflexiva, activa - reflexiva - pragmática, activa reflexiva - teórica, activa - teórica - pragmática y activa - reflexiva - teórica pragmática. De todas ellas, la más significativa fue la activa - pragmática con un 5,7 \%. El estilo Reflexivo apareció asociado con los estilos Teórico y Pragmático en un 8,5 $\%$ con preferencias alta / muy alta. Ampliando las combinaciones afloraron también con el Activo, aunque su representatividad fue menor.

Los estilos Teórico y Pragmático emergieron en una multiplicidad de combinaciones, aunque sus cantidades fueron poco representativas. En total, todas las asociaciones posibles, con preferencias alta / muy alta, aparecían en un 32\% de todos los alumnos encuestados, siendo bastante representativa dicha multiplicidad.

Por género, un $40,46 \%$ de las alumnas presentaron combinaciones de estilos con preferencias altas / muy altas, apareciendo como más abundantes las asociaciones del estilo Activo y las del estilo Reflexivo. En cuanto a las combinaciones de estilos en los alumnos aparecieron en un 36,17\%, mostrándose muy diversificadas. Las más empleadas fueron las asociaciones de los estilos Activo y Reflexivo. Como puede inferirse, las alumnas presentaron valores más altos que los alumnos. 


\subsection{Rendimiento escolar de Física y Química de $4^{\circ}$ de ESO en junio de 2009}

Una vez recogidas las calificaciones escolares en los diferentes Centros y tabulados los datos se obtuvieron los mejores resultados para el Centro 2, lugar en el que se efectuó la intervención, pues fue el que presentó mayores porcentajes de notas altas (sobresaliente y notable) y el que obtuvo menores cantidades de calificaciones bajas (suficiente e insuficiente).

Por género, los alumnos de sexo masculino aprobaron más, suspendieron menos y obtuvieron mejores calificaciones altas que sus homólogas femeninas, aunque las diferencias entre porcentajes no fueran significativas, exceptuando el caso de los insuficientes.

\subsection{Relación entre Rendimiento escolar de Física y Química y preferencias de Estilos de Aprendizaje}

En la muestra constituida por todos los Centros se descubrió que las alumnas con calificación de sobresaliente presentaron fundamentalmente preferencias altas en los estilos Teórico y Pragmático asociados; en cambio, sus homólogos masculinos lo hicieron en los estilos Reflexivo y Teórico, también combinados. Con respecto a las alumnas que obtuvieron calificación de notable, las preferencias se decantaron, fundamentalmente, por las combinaciones de los estilos Reflexivo y Pragmático. Por el contrario, sus compañeros con la misma calificación se orientaron hacia las asociaciones en los estilos Teórico y Pragmático.

Por el contrario, se puso de manifiesto que preferencias baja / muy baja en los estilos Teórico y Reflexivo conducían a un rendimiento escolar insuficiente en alumnas y en alumnos.

\section{CONCLUSIONES}

a)

Aparece una preferencia moderada por todos los Estilos de Aprendizaje en los Centros investigados, aunque un porcentaje significativo de estudiantes presenta preferencias altas b) por combinaciones diversas de los estilos Reflexivo, Teórico y Pragmático.

Rendimientos escolares altos aparecen asociados a preferencias alta / muy alta en los estilos Reflexivo y Teórico. Preferencias escasas en estos estilos se vinculan a rendimientos insuficientes.

c)

El robustecimiento de los diversos estilos mediante estrategias de enseñanza adecuadas conduce a un mayor rendimiento escolar en Física y Química.

\section{REFERENCIAS}


Alonso, Catalina Ma . \& Gallego, D. J. (2003). Tecnología y Educación. Madrid: UNED.

Alonso, Catalina Ma . \& Gallego, D. J. \& Honey, P. (1994). Los Estilos de Aprendizaje. Procedimientos de Diagnóstico y Mejora (1 ${ }^{\mathrm{a}}$. Edición). Bilbao: Mensajero.

Campanario, J. M. \& Moya, A. (1999). Cómo enseñar Ciencias? Principales Tendencias y Propuestas. Enseñanza de las Ciencias, 17 (2):179- 192. Recuperado el 27de Julio de 2011, de http://www2.uah.es/jmc/an11.pdf.

Perales, F. J. \& Cañal de León, P. (2000).Didáctica de las Ciencias Experimentales. Teoría y Práctica de la Enseñanza de las Ciencias .Marfil: Alcoy.

Quintanal Pérez, F. (2011). Estilos de Aprendizaje y Rendimiento Académico en Física y Química. En Gallego, J. \& Alonso C. Innovación y Gestión del Talento (pp. 438 - 448). Cáceres: EBS Business School.

\section{Felipe Quintanal-Pérez}

Doctor en Ciencias de la Educación por la UNED, Licenciado en Ciencias Química por la Universidad Hispalense de Sevilla y Master en Informática Educativa por la UNED. Profesor de Ciencias en Secundaria y Bachillerato, complementándose con diversas tareas realizadas en el ámbito de la educación no formal: campamentos, colonias y albergues con alumnos de Secundaria, Bachillerato, Universidad y colectivos marginales. Ha efectuado publicaciones en revistas, libros y congresos relacionadas con las temáticas de estilos de aprendizaje, TIC y divulgación científica. Como investigador trabaja actualmente en la mejora de los estilos de aprendizaje aplicados a la Física y Química de Bachillerato empleando diversas herramientas Web 2.0. 